\title{
Real-time Stochastic Evacuation Models for Decision Support in Actual Emergencies
}

\author{
ARTURO CUESTA, DANIEL ALVEAR, ORLANDO ABREU and DELFÍN SILIÓ \\ Transports and technology projects and processes \\ University of Cantabria \\ Los castros s/n CP 39005, Santander, Spain
}

\begin{abstract}
This paper introduces and proposes the use of evacuation models for decision support during actual emergencies. Two examples are presented: EvacTrain 2.0 and EvacTunnel. The proposed models are essentially stochastic, quick and easy to use and can generate and process results of several simulations within a few seconds. The main output parameter is the percentile $(0.90,0.95$ or 0.99 th) of total evacuation times. They also provide other statistical characteristics and additional outputs. Both models have been compared with other validated evacuation models. Results suggest that the proposed models provide consistent and reliable results. The general findings described in this paper suggest that it is possible to develop efficient evacuation models for supporting emergency decisions in real-time.
\end{abstract}

KEYWORDS: modelling, real-time, Monte Carlo methods, fire safety management, human behaviour

\section{INTRODUCTION}

In most fire incidents a successful evacuation and rescue can mean the difference between life and death. Therefore, there is a need to predict these processes. Currently, evacuation calculations are becoming a part of fire safety science. In some cases, hand calculations are performed, and in others, modelling and simulation are being used [1]. In the literature, there are some evacuation models reviews. According to Gwynne [2], evacuation models fall into two categories: those which only consider human movement and those which attempt to link movement with behaviour. The first category of evacuation models do not take into account the psychological attributes of people involved in the emergency. The second category of evacuation models are more realistic and take into account the individual behaviour (personal reaction times, exit preference, etc.). According to Tabares [3], the evacuation models are classified into three groups: macroscopic approach, microscopic approach and effect-based simulation approach. In the macroscopic approach, the occupants are modelled as a homogeneous population. In the microscopic approach, occupants are modelled as heterogeneous population (agent based, cellular automata, etc.). In the effect-based approach, the activity based models and those models that incorporate social scientific processes are included. These tools have grown more sophisticated over the time and have been mainly used for performance-based assessments and/or forensic analysis. However, there is an expansion of application opportunities for the evacuation modelling. Gwynne and Kuligowski present a classification of the different application modes: Naïve, Operational, Predictive, Engineered, Interactive and Real-time [4]. These application modes require different levels of data and user expertise. In the real-time mode, the user can acquire feedback from the model during an actual emergency. This requires inputs from the situation to the model which should run significantly faster than real-time. Some evacuation models can run in realtime [5-7]. But, to the authors' knowledge, these models have not been developed specifically for decision support in case of emergency.

This paper presents two evacuation models developed for decision support during actual emergencies. One of the main problems in developing real-time evacuation models is that they are likely to be less sophisticated and produce limited information due to time constraints. The challenge is to obtain equilibrium between run times and providing enough detail in the model to allow sufficient accuracy. To address this, Monte Carlo methods can be used varying the key random parameters in order to capture all the possible situations in which a given scenario might be evacuated. This means generating representative and significant samples of outputs variables. The real-time applications require processing the outputs quickly enough and the information provided need to be easy to interpret and with a high confidence level. 
For instance, given a scenario, the main output parameter may be the percentile (90, 95 or 99 th) of total evacuation times. The evacuation models presented in this paper operate in the manner described above.

\section{EVACTRAIN 2.0}

\section{Motivation}

Fire incidents inside passenger trains can constitute a significant risk to life. The train crew are responsible for passenger safety during an on-board fire emergency. The first priority is to direct passengers away from fire and inform the train operations centre about the situation. The second priority is to determine when and how perform the evacuation. For instance, whether to reach an appropriate place for the evacuation (i.e., the closest station, platform) or stop the train as soon as possible and perform evacuation to the tracks. Furthermore, the crew needs to know the number of passengers on-board, the dangers present inside and outside the train, a safe area where passengers should be moved and which doors should be opened. Therefore, it is not easy to make the correct decisions. The use of computer modelling analyses in real-time could improve such decisions under a variety of emergency conditions.

\section{Overview of the model}

EvacTrain 2.0 is an object-oriented evacuation model developed by GIDAI Group. The purpose of the model is to simulate different evacuation strategies in trains. The train spaces are represented by a coarse network. Each node represents a passenger coach with exits. Due to the fact that EvacTrain 2.0 is a model tailored to the intended train, data related to the train characteristics are included by default in the model. The model considers the following basic scenarios:

1. Emergency evacuation to platform (tunnel).

2. Emergency evacuation to track level.

3. Evacuation to platform.

4. Evacuation to track level.

5. Evacuation to other train.

Evacuation scenarios 3-5 are not considered as emergency situations. Therefore, they are excluded from the present description. It is assumed that passengers are ready to start evacuation once the train stops. However, the model includes the preparation time. This is a random variable defined as the time elapsed from the stop of the train until passengers start evacuation. The values vary according to the emergency evacuation scenario. In evacuation to platform, this variable is the time spent in opening he train doors. In evacuation to track level, this variable represents the time spent to set up the portable ladders or ramps. EvacTrain 2.0 focuses on the simulation of the flow through the available exits. The model considers the flow as a random variable individually assigned to each passenger from normal distributions. The values of the distributions vary according to the different exit conditions (to platform, directly to track and through emergency ladders). The values used by default in the model are obtained from [8-10]. It should be noted that the model is flexible and allows the user to modify these data.

Some exits may be unavailable in cases of evacuation. The user has the option to block the exits (or to set up the available exits for evacuation) in order to reproduce the real situation or to explore the potential outcomes of an evacuation strategy. In these cases, the model simulates the relocation process providing a realistic distribution of the number of passengers at each exit. Then the critical exit is defined as the exit used by the maximum number of passengers.

The model performs 250 iterations by default. The evacuation time of $i$-th iteration is given by:

$$
t_{e_{i}}=t_{p_{i}}^{(k)}+\left.t_{c r i t_{i}}^{(k)}\right|_{i=1, n_{i t e r}}
$$

Where:

$\begin{array}{cl}k & \text { - The evacuation scenario (emergency evacuations to platform or to track level); } \\ t_{p_{i}}^{(k)} & \text { - Preparation time for the } k \text { evacuation scenario and i-th iteration; }\end{array}$ 
$t_{\text {crit }_{i}}^{(k)} \quad$ - Critical exit time:

$$
t_{c r i t_{i}}^{(k)}=\sum_{j=1}^{m_{p a s}} \frac{1}{f_{j}^{(k)}}
$$

Where:

$m_{\text {pas }} \quad$ - Number of passengers that use the critical exit;

$f_{j}^{(k)} \quad$ - Random flow assigned to $j$-th passenger according to the evacuation conditions $(k)$.

Due to its intended use, the presented model is quick and easy to set-up. The input parameters consist of:

- Fraction of the full load: value between 0 and 1 known by the train crew.

- Type of incident: 1) fire, 2) collision, 3) derailment, 4) technical failure.

- Evacuation destination: 1) platform, 2) track level or 3) other train (only for transfers).

- Number of available exits.

EvacTrain 2.0 generates and process results in a few seconds. The outputs produced by the model are displayed at the screen and they can be saved in txt files as well. The model statistically treats the sample of total evacuation times and fits it to a known distribution (if possible). Otherwise, density estimations are given. The main output parameter is a percentile of egress times $(0.90,0.95$ and 0.99 th). It also provides other statistical characteristics: mean, variance, maximum and minimum values. Additional outputs include the number of available exits, the critical exit and the number of passengers that use it.

\section{Comparison with STEPS}

In this section we describe a detailed comparison between EvacTrain 2.0 and STEPS model [11]. As explained above, this is a model tailored to the intended train. The comparison presented here is performed for a high speed train S 102. This is a train $200 \mathrm{~m}$ length with 11 passenger coaches $(+1$ lounge) and capacity for 316 passengers. Two sets of evacuation scenarios are considered: emergency evacuation to platform and emergency evacuation to track level. Figure 1 shows the emergency evacuation scenarios to platform.

\section{Scenario 0}

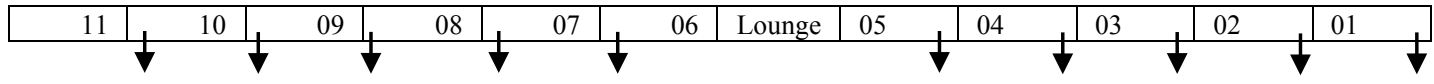

Scenario $3 \mathrm{t}$

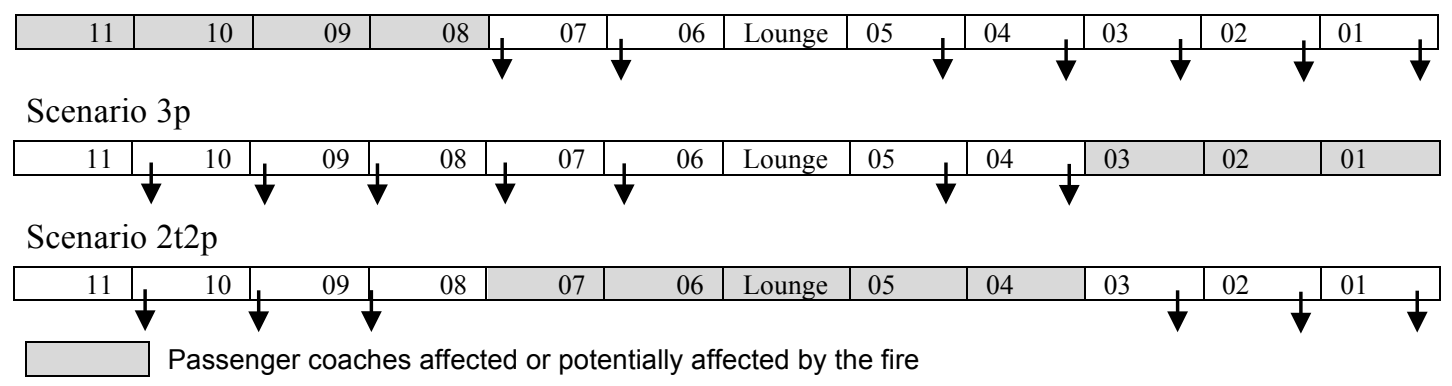

Fig. 1. Emergency evacuation scenarios to platform. 
In emergency evacuation scenarios to platform, two consecutive dynamic processes have to be simulated: 1) evacuating passengers to a place of relative safety along the train (pre-evacuation activities) and 2) evacuating from the train. EvacTrain 2.0 simulates the relocation process to provide a realistic distribution of the number of passengers at each exit.

On the other hand, STEPS allows changing the availability of certain exits during the course of the simulation by using "exit events". Using this feature, the user can open, close or make exits unavailable. When an exit is set to closed, the agents will still consider the exit when choosing their target and form a queue in front of it. When the exit is unavailable, it is considered to be no longer usable, and nobody moves towards that exit.

Table 1 displays the inputs considered for the comparison of the emergency evacuation scenarios to platform. The values are obtained from an announced evacuation drill [8].

Table 1. Inputs for the comparison of emergency evacuation to platform.

\begin{tabular}{|l|c|c|}
\hline \multicolumn{1}{|c|}{ Inputs } & EvacTrain 2.0 & STEPS \\
\hline N of passengers & 316 & 316 \\
\hline Flow through the exits (per/s) & $0.44 \pm 0.20$ & 0.44 \\
\hline Walking speed (m/s) & Not considered & $0.99 \pm 0.20$ \\
\hline Time to open the doors (s) & 35 & 35 \\
\hline
\end{tabular}

Scenario $1 \mathrm{~b}$ and Scenario $2 \mathrm{~b}$ in Figure 2 represent emergencies in which passengers have to evacuate to the track level by using 1 and 2 emergency ladders respectively. Table 2 displays the inputs considered for the comparison of the emergency evacuation scenarios to track level. The emergency ladders are $3 \mathrm{~m}$ long and consist of two separate parts that have to be assembled. These evacuation elements hold two passengers simultaneously. It is considered an average time of $3 \mathrm{~s}$ spent by each passenger to negotiate the emergency ladder (flow of $0.33 \mathrm{per} / \mathrm{s}$ ). This value is derived from Volpe Center egress trials to track level $[12,13]$. The preparation time is a random parameter in EvacTrain 2.0. However, it is set as a constant value of $300 \mathrm{~s}$ to represent the same conditions in both models.

Scenario $1 b$

\begin{tabular}{|l|l|l|l|l|l|l|l|l|l|l|l|}
\hline 11 & 10 & 09 & 08 & 07 & 06 & Lounge & 05 & 04 & 03 & 02 & 01 \\
\hline
\end{tabular}

Scenario $2 b$

\begin{tabular}{|l|l|l|l|l|ll|l|l|l|l|l|l|}
\hline 11 & 10 & 09 & 08 & 07 & 06 & Lounge & 05 & 04 & 03 & 02 & 01 \\
\hline
\end{tabular}

Fig. 2. Emergency evacuation scenarios to track level trough emergency ladders.

Table 2. Inputs for the comparison of evacuation to track level.

\begin{tabular}{|c|c|c|}
\hline Inputs & EvacTrain 2.0 & STEPS \\
\hline Occupation $100 \%$ & 316 & 316 \\
\hline Flow through the exits (per/s) & $0.33 \pm 0.10$ & 0.33 \\
\hline Walking speed $(\mathrm{m} / \mathrm{s})$ & Not considered & $(0.99 \pm 0.20) * 0.80$ \\
\hline Time to install the emergency ladder (s) & 300 & 300 \\
\hline
\end{tabular}

As mentioned previously, in EvacTrain, passengers are ready for evacuation once the train stops. The model focuses on the simulation of exit performance and the flow is represented as a random time spent by 
each passenger to negotiate the exit. Therefore, as Tables 1 and 2 show, walking speed is not included as an input variable in the proposed model.

Each scenario was run 100 times. It is assumed that the passengers are compliant with the train crew's commands. This assumption is a basic requirement for seeing the effects of the procedures that are implemented. For the evacuation to the track level, no alternative escape routes, such as other exit doors where the passengers have to climb higher than $1.1 \mathrm{~m}$, are considered.

Figures 3 and 4 show the cumulative distribution functions of total evacuation times. The statistical characteristics are shown in Tables 3 and 4. It is possible to see a wider variability on the sample provided by EvacTrain 2.0 in Scenario 1b (see in Figure 4). This is because of the random flow in the single exit simulated by the proposed model, not reproduced by STEPS model which uses a constant value. However, there is a good agreement between both models. This is quantified by the Percent Error (PE) of the mean (PEM) and the 95 th percentile (PEP) of total evacuation times. Note that the results of the proposed model are considered as the "approximate" values while the results of model of the comparison are considered as the "actual" values. In the emergency evacuation to platform both the PEM and PEP are lower than 3\%. Furthermore, in the emergency evacuation to track level scenarios, the PEM and PEP are lower than $1.3 \%$. Based on these results, it can be argued that EvacTrain is capable of producing reliable predictions of total evacuation times.
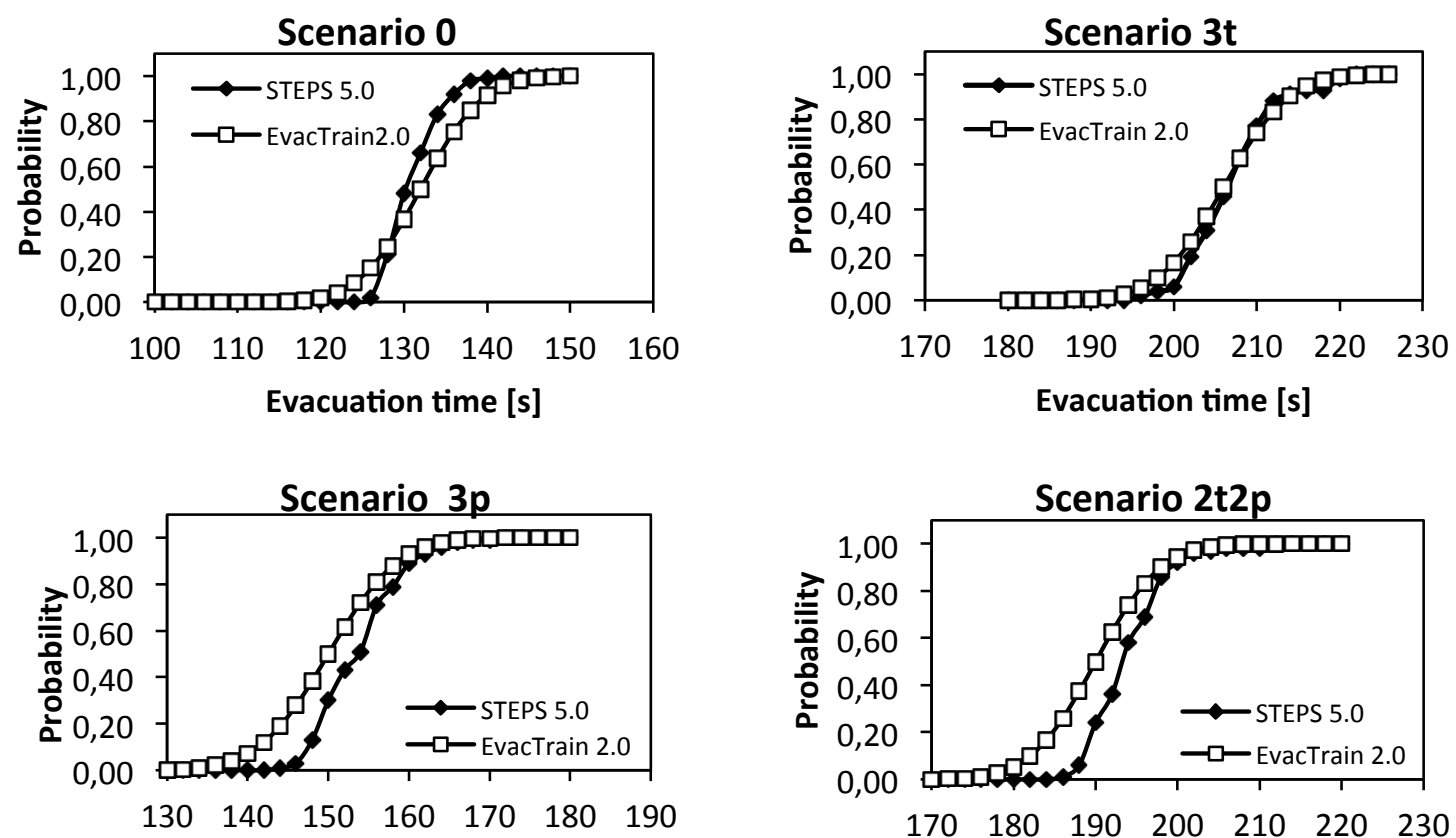

Evacuation time [s]

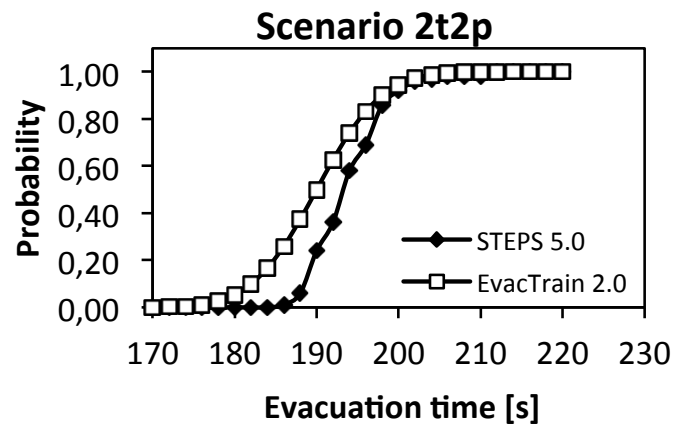

Fig. 3. Cumulative distribution functions of the total evacuation times to platform.

Table 3. Distributions of the total evacuation times to platform (s).

\begin{tabular}{|l|c|c|c|c|c|c|c|c|}
\hline \multirow{2}{*}{ Scenario } & \multicolumn{4}{|c|}{ EvacTrain 2.0 } & \multicolumn{4}{c|}{ STEPS 5.0 } \\
\cline { 2 - 9 } & Mean & S.D. & Range & Perc. 95th & Mean & S.D. & Range & Perc. 95th \\
\hline Scenario 0 & 132 & 5.8 & $116-151$ & 143 & 131 & 3.2 & $126-142$ & 139 \\
\hline Scenario 3t & 206 & 6.2 & $183-220$ & 217 & 207 & 5.4 & $195-222$ & 214 \\
\hline Scenario 3p & 150 & 6.8 & $130-171$ & 160 & 154 & 5.3 & $144-171$ & 161 \\
\hline Scenario 2t2p & 190 & 6.2 & $169-206$ & 200 & 194 & 4.7 & $186-212$ & 205 \\
\hline
\end{tabular}



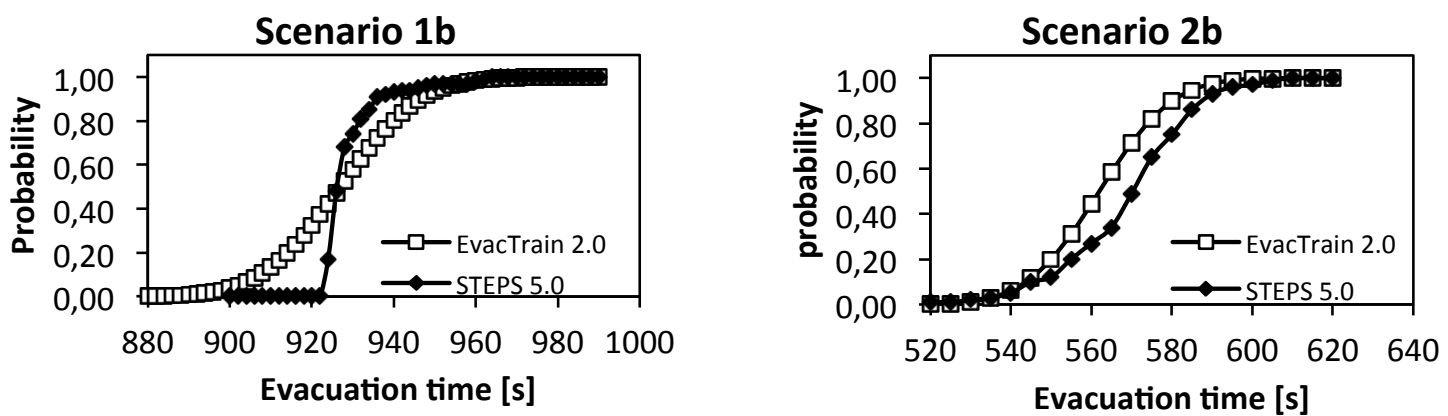

Fig. 4. Cumulative distribution functions of total evacuation times to track level.

Table 4. Distributions of the total evacuation times to track level (s).

\begin{tabular}{|c|c|c|c|c|c|c|c|c|}
\hline \multirow{2}{*}{ Scenario } & \multicolumn{4}{|c|}{ EvacTrain 2.0 } & \multicolumn{4}{c|}{ STEPS 5.0 } \\
\cline { 2 - 9 } & Mean & S.D. & Range & Perc. 95th & Mean & S.D. & Range & Perc. 95th \\
\hline Scenario 1b & 927 & 15.3 & $878-970$ & 950 & 929 & 9.5 & $922-984$ & 939 \\
\hline Scenario 2b & 562 & 14.2 & $512-597$ & 585 & 569 & 16.3 & $519-606$ & 590 \\
\hline
\end{tabular}

\section{EVACTUNNEL}

\section{Motivation}

Road tunnels constitute dangerous environments when a fire occurs. Past disasters have shown the need for an effective emergency response and the tragic consequences of incorrect or delayed decision making [14]. The tunnel operator is the first person to deal with the emergency. He/she detects the emergency mainly through the Automatic Incident Detection (AID) system and/or CCTV. But, the information may be sparse, incomplete and inaccurate and he/she will be required to make decisions such as closing the tunnel, declaring the evacuation, etc. In many cases, these decisions are based on fixed protocols and can be made too late. The use of predictive tools to support the operator decisions in real-time could improve road tunnel safety. In this sense, the GIDAI Group has developed a Decision Support System (DSS) for emergency management in road tunnels [15]. The DSS analyses the current situation and guides the course of decisions to deal with the emergency. Furthermore, the system provides real-time estimation of the severity of the accident and the required evacuation and rescue times by the evacuation model integrated in the system: EvacTunnel.

\section{Overview of the model}

EvacTunnel is an object-oriented evacuation model developed by GIDAI Group [16]. The purpose of the model is to simulate the evacuation and rescue processes in road tunnels. As Figure 5 shows, the presented model considers two areas inside the tunnel. The Area 1 includes the vehicles and the people directly involved in the accident, where it is likely to find injured people who cannot evacuate by themselves (rescue process). The Area 2 includes the vehicles and people trapped inside the tunnel not directly affected by the accident. This people can leave the tunnel by themselves (self-evacuation process). The model calculates both scenarios separately.

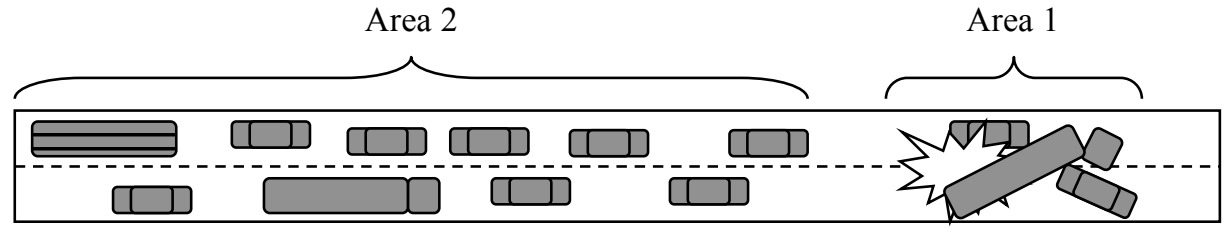

Fig. 5. Areas inside the tunnel considered in the model.

The model allows performing several simulations (a minimum of 100 runs). In each simulation, the model registers the evacuation time for all tunnel users and considers the time when the last one leaves the tunnel. 
This is calculated for Area 1 and Area 2 separately. The evacuation time of each tunnel user depends upon the pre-movement time, unrestricted walking speed and the distance through the escape routes:

$$
t_{e_{i}}=t_{p m_{i}}+\frac{d_{\text {mov }_{i}}}{v_{\text {mov }_{i}}}
$$

Where:

$t_{e_{i}} \quad$ - Evacuation time for the i-th person;

$t_{p m_{i}} \quad$ - Pre-movement time for the i-th person;

$d_{\text {mov }_{i}} \quad$ - Distance to the exit for the i-th person;

$v_{\text {mov }_{i}} \quad$ - Walking speed for the i-th person.

For people in Area 1, who cannot evacuate by themselves (assisted mobility), the pre-movement time $\left(t_{p m_{i}}\right)$ is calculated by the following expression:

$$
t_{p m}^{(M A)}=t_{\text {not }}+t_{\text {react }}+t_{\text {mov }}+t_{\text {exam }}
$$

Where:

$M A \quad$ - Assisted Mobility. People who cannot evacuate by themselves;

$t_{\text {not }} \quad$ - Delay time to inform the emergency services;

$t_{\text {react }}$ - Reaction time of emergency services;

$t_{\text {mov }} \quad$ - Travelling time to arrive to "the scene";

$t_{\text {exam }}$ - Time to examine and prepare the users affected by the accident.

In Area 2, the pre-movement time $\left(t_{p m_{i}}\right)$ of the people trapped inside the tunnel can be divided into two phases:

1) Recognition Phase $\left(t_{p m_{i j}}^{(1)}\right)$.- The time required to understand what has happened.

2) Response Phase $\left(t_{p m_{i j}}^{(2)}\right)$.- The time spent to leave the vehicle and start evacuation movement.

The model implements the pre-movement time by using the criterion of distance from the accident. Figure 6 shows the Recognition Phase $t_{p m_{i j}}^{(1)}$ as linearly dependent with the distance respect to the accident zone. The model calculates the Recognition Phase considering the time needed by the persons next to the accident area to reach different locations during their movement towards the exit with a speed of $1.55 \mathrm{~m} / \mathrm{s}$. This walking speed can be changed by the user of the model. 


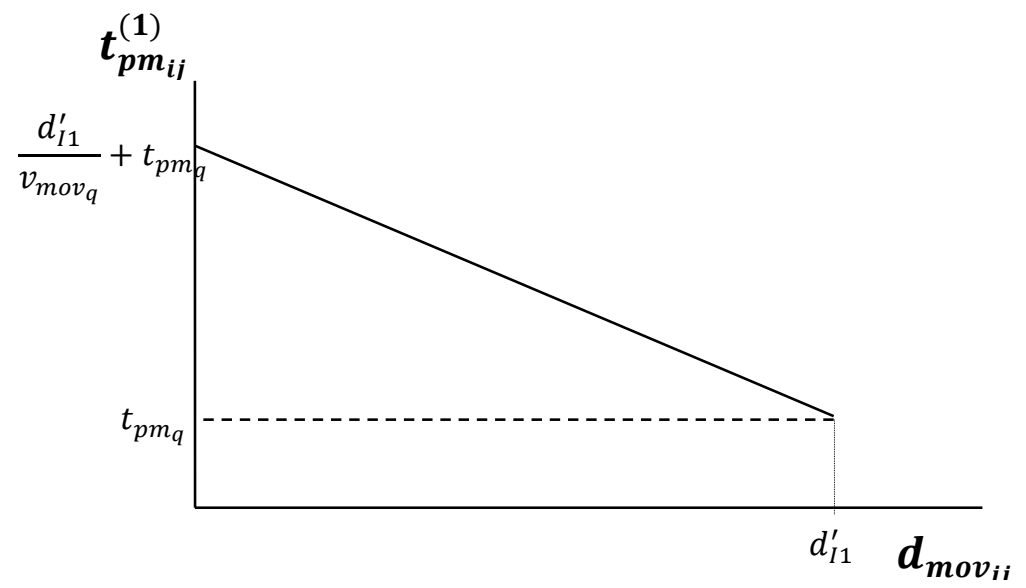

Fig. 6. Linearly dependence of $t_{p m_{i j}}^{(1)}$ with the distance.

Therefore the Recognition Phase of tunnel users is:

$$
t_{p m_{i j}}^{(1)}=t_{p m_{q}}+\frac{d_{I 1}^{\prime}-d_{\text {mov }_{i j}}}{v_{\text {mov }_{q}}}
$$

Where:

$t_{p m_{q}} \quad$ - Pre-movement time of the first person to respond near to the accident (constant value);

$d_{I 1}^{\prime} \quad$ - Travelling distance of Area 2;

$d_{\text {mov }_{i j}}$ - Travelling distance to each tunnel user;

$v_{\text {mov }_{q}} \quad$ - Unimpeded walking speed of the first person to respond near the accident.

The theoretical distribution for the Response Phase $t_{p m_{i j}}^{(2)}$ is derived from an experiment conducted at University of Cantabria, Spain. The time spent by 32 participants to leave their vehicles was measured. The participants spent between $15 \mathrm{~s}$ and $120 \mathrm{~s}$. The distribution has a mean of $67.5 \mathrm{~s}$ and a standard deviation of $17.5 \mathrm{~s}$. Default walking speeds are assigned from a normal distribution with a mean of $1.25 \mathrm{~m} / \mathrm{s}$ and a standard deviation of $0.32 \mathrm{~m} / \mathrm{s}$. These values are derived from [17].

The real-time mode requires a direct observation from the real situation (i.e. through CCTV) and then provides this information directly to the evacuation model. However, during the first stages of the emergency, there is a high level of uncertainty regarding the number of vehicles and the number and distribution of occupants. The number and characteristics of people involved in the accident (Area 1) is predicted by the Incidents Model integrated in the DSS [15]. In this first version of the model, the tunnel operator introduces an estimation of the number of vehicles in Area 2. Otherwise, this information can be obtained from the traffic counters in the tunnel. The number of people inside the vehicles is a random variable between a maximum and minimum value that can be predefined by the user (i.e 1-5 occupants/car, 1-2 occupants/truck and 20-40 occupants/bus). Therefore, in each simulation the occupation load is different.

Taking into account that all the variables listed above are random variables, their generation using Monte Carlo methods can be represented analytically by using the inverse transformation method (Smirnov transform) [18]. Although in particular cases, other algorithms can be used, such as the Box-Muller for normal and lognormal distributions, or the numerical integration method for estimating the distribution function by a histogram. 
EvacTunnel statistically treats the sample of total evacuation times and fit it to a known distribution (if possible). Otherwise, density estimation is given using histogram. The main output parameter is a percentile of evacuation times $(0.90,0.95$ and 0.99$)$. The model also provides other statistical characteristics: mean, variance, maximum and minimum values. Additional outputs include the number of people trapped inside the tunnel at specific time and location.

\section{Comparison with STEPS, Pathfinder and GridFlow}

Here we describe the comparison analysis between EvacTunnel and other current evacuation models: STEPS [11], Pathfinder [19] and GridFlow [20]. The comparison is performed for the self-evacuation of people trapped inside the tunnel. Figure 7 shows the layout of the evacuation scenario for the simulations. It consists of an accident in the center of the tube obstructing the access to the cross passage. The evacuation is modelled considering the moment in which the vehicles are stopped, queuing behind the vehicles involved in the accident. It is assumed a total of 54 vehicles trapped in the tunnel: 49 light vehicles (cars) and 5 heavy vehicles (trucks). The occupation load is assumed to be 1 person per heavy vehicle. For light vehicles a load factor of 2.32 is considered. Therefore, 119 occupants are considered for the simulations.

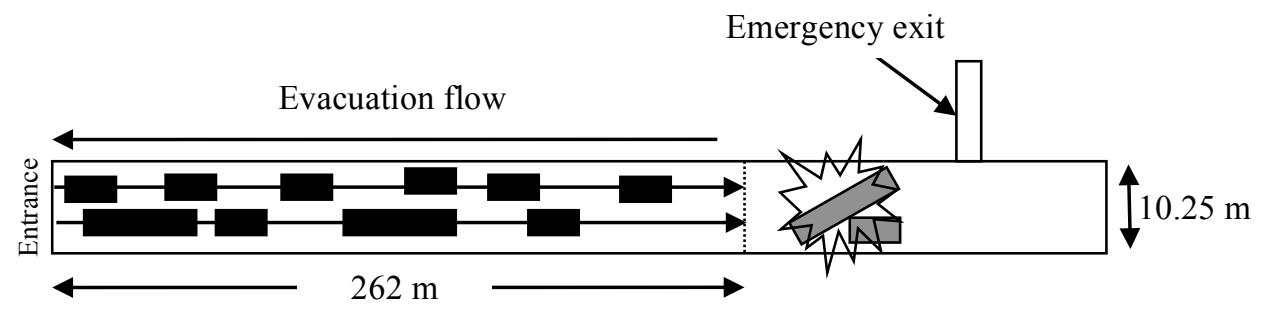

Fig. 7. Layout of the evacuation scenario considered for simulations.

Two tests are considered. In Test 1, no behaviour is performed in order to check that the simulation of movement is working satisfactorily. In Test 2 a behaviour comparison is performed.

Table 5 displays the evacuation times obtained in Test 1 . The evacuation times are very similar between the models. The Percent Error (PE) is not higher than $1 \%$ between the proposed model and the models of the comparison. The small differences are found due to the random distribution of the occupants who are further from the tunnel portal (their start position). Results from Test 1 show that basic movement components of EvacTunnel work adequately.

Table 5. Results of Test 1 (s): 1 run.

\begin{tabular}{|c|c|}
\hline Model & Evacuation time \\
\hline Pathfinder & 260 \\
\hline STEPS & 257 \\
\hline GridFlow & 258 \\
\hline EvacTunnel & 262 \\
\hline
\end{tabular}

Test 2 provides an opportunity to validate the proposed model against other evacuation models. In this test the scenario has been run 100 times to capture stochastic variations in the results. The implementation of pre-evacuation times in GridFlow, STEPS and Pathfinder models has been done using the criterion of distance from the accident as it is considered by the proposed model. In this way a phased response of the occupants has been considered. In order to implement this, the tube was divided into 13 zones $(20 \mathrm{~m}$ length) with different population groups and pre-movement time distributions. In GridFlow these zones were implemented by different spaces connected by links (inlet and outlet). In STEPS this was done by using locations on the plane. In Pathfinder rectangular rooms were used. The pre-movement times have been assigned using normal distribution laws. In Zone 1 it was assumed a pre-movement time distribution with a mean of $170 \mathrm{~s}$ and a standard deviation of $17.5 \mathrm{~s}$. Then, the mean value has been increased by $13 \mathrm{~s}$ per zone in order to reproduce the same "domino effect" applied by the proposed model. The same 
unimpeded walking speed distribution has been assigned for all occupants in all models. This is a normal distribution with a mean value of $1.20 \mathrm{~m} / \mathrm{s}$ and a standard deviation of $0.20 \mathrm{~m} / \mathrm{s}$.

Figure 8 shows the cumulative distribution functions of evacuation times and Table 6 displays a comparison of the mean, maximum, minimum and 95 th percentile of total evacuation times obtained by the models. The predicted evacuation times do not vary significantly among each model and their curves are very similar. In this case the evacuation time was driven by the interactions between pre-movement time and the travel distributions. Table 7 shows the PEM and PEP when EvacTunnel is compared with the other models. The PEM is lower than $1.5 \%$. The maximum PEP is $5.62 \%$ when the proposed model is compared with Pathfinder. The results from Test 2 show that EvacTunnel is able to provide as reliable predictions as the models of the comparison.

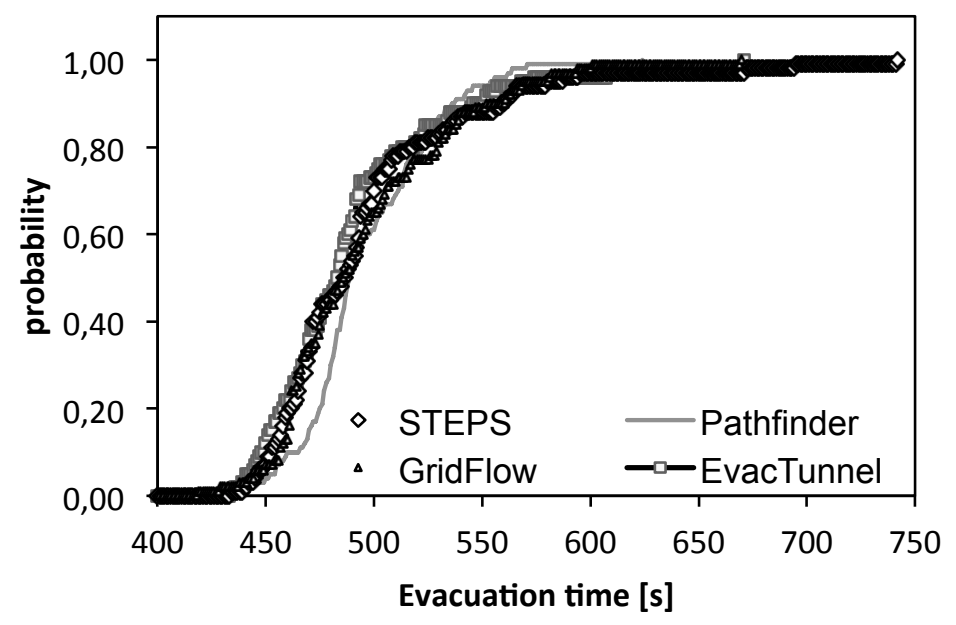

Fig. 8. Cumulative distribution functions of total evacuation times.

Table 6. Results of Test 2 (s): 100 runs.

\begin{tabular}{|l|c|c|c|c|}
\hline & Pathfinder & STEPS & GridFlow & EvacTunnel \\
\hline Mean & 497 & 496 & 495 & 491 \\
\hline S.D. & 31 & 50 & 42 & 44 \\
\hline Range & $429-624$ & $434-742$ & $419-670$ & $434-671$ \\
\hline Perc. 95th & 554 & 580 & 570 & 587 \\
\hline
\end{tabular}

Table 7. Percent Errors of Test 2 when EvacTunnel is compared with other models.

\begin{tabular}{|c|c|c|}
\hline Model & PEM (\%) & PEP (\%) \\
\hline Pathfinder & 1.22 & 5.62 \\
\hline STEPS & 1.02 & 1.19 \\
\hline GridFlow & 0.81 & 2.90 \\
\hline
\end{tabular}

\section{DISCUSSION OF THE RESULTS}

Making decisions is certainly the most important task of a safety manager (operator) and it is often a very difficult one. The use of evacuation models for decision support involves the following basic requirements: 1) providing enough detail in the model to allow sufficient accuracy and 2) fast simulation times. 
Firstly, a good decision should not be supported by an outcome alone. Deterministic models are likely to produce an inaccurate representation of the evacuation process as they only consider one or a few potential situations. This is due to the uncertainty related to the situation and the uncertainty related to the human behavior during evacuation process. The use of Monte Carlo methods permits the representation of all possible situations and the generation of samples of total evacuation times. The stochastic simulations generate more reliable and consistent results. Furthermore, the use of distributions of the total evacuation times provides more powerful criteria for decision making. For instance, the use of percentiles $(90,95$ or 99 th).

Secondly, real-time evacuation models have to be a simplified representation of the actual situation. Therefore, they should concentrate in the most essential parameters and interactions and ignores the less essential ones. The proposed models are based on the idea that evacuation calculations can be performed by addressing a small set of random parameters that have impact in the outcomes [21]. For the analysis of evacuation process in passenger trains, we suggest that the dominant parameters are the time spent to prepare for evacuation and the flow through the available exits. The first parameter involves specific evacuation procedures such installing the emergency ladder. The second parameter depends on the evacuation conditions defined by the number of passengers per available exit and the evacuation destination (platform, track level). For the analysis of evacuation process in road tunnels, we suggest that the dominant parameters are the pre-movement times, the walking speeds and the travelling distances of individuals.

Clearly, the results of the comparison suggest that the additional complexity of the current evacuation models may not yield significantly different results than the proposed models. In train evacuation scenarios, both the PEM and the PEP are lower than 3\% when EvacTrain is compared with STEPS model. In road tunnel evacuation scenarios, the PEM is not higher than $1.22 \%$ when EvacTunnel is compared with other three evacuation models (Pathfinder, STEPS and GridFlow). Furthermore, the PEP is not higher than the $5.62 \%$ when EvacTunnel is compared with Pathfinder. Therefore, it can be argued that the presented models provide consistent and reasonable results.

Tables 8 and 9 show a comparison of the simulation times and capabilities between the evacuation models used for this study. The information provided is based on our experiences and estimations for 100 runs, once the scenarios have been implemented (geometry, number of occupants, etc.). From the Tables 8 and 9 it is possible to see that the proposed models can perform several simulations and process the results statistically by themselves in a few seconds.

Table 8. Comparison of simulation times and capabilities for train evacuation analyses.

\begin{tabular}{|c|c|c|c|c|}
\hline Model & Batch run? & $\begin{array}{c}\text { Batch run time } \\
\text { (100 runs) }\end{array}$ & $\begin{array}{c}\text { Plotting } \\
\text { data? }\end{array}$ & $\begin{array}{c}\text { Statistical } \\
\text { processing? }\end{array}$ \\
\hline EvacTrain 2.0 & Yes & $<5 \mathrm{~s}$ & Yes & Yes \\
\hline STEPS & Yes & $\begin{array}{c}70-130 \mathrm{~s} \text { (Evacuation } \\
\text { to platform) } \\
210-390 \mathrm{~s} \text { (Evacuation } \\
\text { to track level) }\end{array}$ & No & No \\
\hline
\end{tabular}

Table 9. Comparison of simulation times and capabilities for road tunnel evacuation analyses.

\begin{tabular}{|c|c|c|c|c|}
\hline Model & Batch run? & $\begin{array}{c}\text { Batch run time } \\
\text { (100 runs) }\end{array}$ & $\begin{array}{c}\text { Plotting } \\
\text { data? }\end{array}$ & $\begin{array}{c}\text { Statistical } \\
\text { processing? }\end{array}$ \\
\hline EvacTunnel & Yes & $<5 \mathrm{~s}$ & Yes & Yes \\
\hline STEPS & Yes & $400 \mathrm{~s}$ & No & No \\
\hline Pathfinder & No & $>3600 \mathrm{~s}$ & No & No \\
\hline GridFlow & Yes & $403 \mathrm{~s}$ & Yes & No \\
\hline
\end{tabular}


These real-time evacuation models are appropriate and accurate in specific situations. They can be used to make critical decisions during the first stages of the emergency. In EvacTrain 2.0 the user can explore different evacuation processes and choose and appropriate evacuation strategy. For instance, if a fire is detected in the running train whether to stop as soon as possible or reach a platform for the evacuation. EvacTunnel enables the user to overcome the uncertainty during the first stages of the emergency. For instance, the tunnel operator can declare the evacuation based on the model predictions, as a preventive strategy, or inform about the required times for evacuation to emergency services before their arrival to the "scene".

\section{CONCLUSIONS}

From this work it is concluded that evacuation calculations can be used for supporting timely decisions during actual emergencies. It is proposed the use of stochastic models with the capability to perform several simulations by changing key random parameters to capture all potential outcomes. These models should process the results by themselves and provide information easy to interpret for decision making. All this process should be performed within a few seconds. Two evacuation models which operate in the manner describe above have been presented and partially validated against other evacuation models. Note that the evacuation models presented here can also be used for other applications such as performance-based assessments and/or risk analysis.

Most of the input parameters included in the models are obtained from empirical research. However, it should be noted that the flexibility of the models allows the user to change these values. Therefore, it is recommended as a good practice to use reliable data from trials and/or evacuation drills in the scenarios where the evacuation models are going to be implemented.

The current versions of the proposed models have limitations and new challenges to be addressed. Future research will include further validation against experiments and evacuation drills for a set of new possible scenarios.

\section{ACKNOWLEDGMENTS}

The authors would like to thank to the Spanish Ministry of Economy and Competitiveness for the EVACTRAIN Project grant, Ref.: BIA2011-26738, co financed by FEDER funds. 


\section{REFERENCES}

[1] Kuligowski, E. D., "Modeling Human Behavior During Building Fires”, NIST National Institute of Standards and Technology, NIST Technical Note 1619, USA, December, 2008.

[2] Gwynne, S., et al., (1999) A Review of the Methodologies Used in the Computer Simulation of Evacuation from Building Environment, Building and Environment 34, 741-749.

[3] Tabares, R. M., (2009) Evacuation Process Versus Evacuation Models: “Quo Vadimus?”, Fire Technology 45, 419-430.

[4] Gwynne, S., and Kuligowski, E., "Application Modes of Egress Simulations”, Proceedings of the $4^{\text {th }}$ International Conference Pedestrian and Evacuation Dynamics, Springer, 2008, pp.397-409.

[5] Kisko, T.M, and Francis, R.L., (1985) Evacnet+: a computer program to determine optimal evacuation plans, Fire Safety Journal 9, 211-220.

[6] Lin, Y., et al., "Agent-Based Simulation of Evacuation: An Office Building Case Study", Proceedings of the $4^{\text {th }}$ International Conference Pedestrian and Evacuation Dynamics, Springer, 2008, pp.345-357.

[7] Yamashita, T., et al. "Exhaustive testing plan with high speed evacuation simulator", Proceedings of the International Scientific Conference Emergency Evacuation of People from Buildings, 2011, pp.357-363.

[8] Capote, J. A., Alvear, D. M., Abreu, O. V., Cuesta, A. and Alonso, V., (2012) A Stochastic Approach for Simulation Human Behavior during Evacuation Process in Passenger Trains, Fire Technology 48: 911-925.

[9] Capote, J. A., Alvear, D. M., Abreu, O. V. and Cuesta, A., (2012) Analysis of evacuation procedures in high speed trains fires, Fire Safety Journal 49:35-46.

[10] Norén, A., and Winér, J., "Modelling Crowd Evacuation from Road and Train Tunnels-Data and design for faster evacuations", Report 5127, Department of Fire Safety Engineering Lund University, Sweden, 2003.

[11] STEPS Simulation of Transient and Pedestrian movementS: User Manual, unpublished, available with egress model from Mott MacDonald. http://www.mottmac.com.

[12] Markos, S.H, and Pollard, J.K. "Passenger Train Systems: Single-Level Commuter Rail Car Egress Experiments", Prepared by Volpe Center/USDOT for FRA/USDOT. Final Report. In FRA report approval process as of May 2013.

[13] Galea, R.E., et al. "The Developement and Validation of a Rail Car Evacuation Model", Proceedings of the $13^{\text {th }}$ International Fire Science \& Engineering Conference INTERFLAM 2013, Interscience, 20013, pp.1013-1034.

[14] Carvel, R., and Marlin, G., "A history of fire incidents in tunnels", The Handbook of Tunnel Fire Safety, Alan Beard and Richard Carvel, UK, 2005, p. 3-37.

[15] Alvear, D., Abreu, O., Cuesta, A., and Alonso, V., (2013) Decision support system for emergency management: Road tunnels, Tunnelling and Underground Space Technology 34, 13-21.

[16] Capote, A., Alvear, D., Abreu, O., Cuesta, A., and Alonso, V., (2013) A real-time stochastic evacuation model for Road tunnels, Safety Science 52, 73-80.

[17] Boyce, K.E., Shields, T.J, and Silcock, G.W.H., (1999) Toward the Characterization of Building Occupancies for Fire Safety Engineering: Prevalence, Type, and Mobility of Disabled People, Fire Technology, 35, 1, 35-50.

[18] Rubinstein, R.Y., Kroese, D.P., Random Number, Random Variable and Stochastic Process Generation. Simulation and Monte Carlo Method, 2012, Wiley-Interscience, pp. 49-80. 
[19] Thorton, C., O'Konski, R. and Hardeman, B., "Introducing -: An Agent-Based Egress Simulator", Proceedings of the Fourth International Symposium on Human Behaviour in Fire, UK, 2009, pp.567-572.

[20] Bensilium, M. and Purser. D., "GridFlow: an Object-Oriented Building Evacuation Model Combining Pre-movement and Movement Behaviours for Performance-Based Design", Proceedings of the Seventh International Symposium on Fire Safety Science, USA, 2003, pp. 941952.

[21] Purser, D., "Dependence of Modelled Evacuation Times on Key Parameters and Interactions", Proceedings of the Ninth International Symposium on Fire Safety Science, Germany, 2003, pp. 353-364. 